\title{
Regeneración ósea guiada simultánea en implantes cigomáticos con una aproximación exteriorizada en una atrofia maxilar avanzada
}

\section{Guided bone regeneration and zygomatic implants: a case report}

\author{
M. Peñarrocha Diago*, J. Aizcorbe Vicente**, M. Díaz Sánchez**, \\ B. Serra Pastor***, D. Soto-Peñaloza**, D. Peñarrocha Oltra****
}

\section{RESUMEN}

Se presenta un caso clínico en el que se rehabilita a una paciente con maxilar superior atrófico mediante una combinación de implantes cigomáticos, colocados mediante una aproximación exteriorizada, e implantes anteriores convencionales palatinizados. Se realizó un procedimiento de regeneración ósea guiada alrededor de los implantes cigomáticos para obtener un mayor grosor de la cortical vestibular y palatina alrededor de los implantes del maxilar superior y mejorar el pronóstico. A un año de la carga protésica, el hueso periimplantario se mantiene estable.

PALABRAS CLAVE: Implantes cigomáticos, regeneración ósea guiada, arco completo, maxilar atrófico, prótesis fija.

\section{ABSTRACT}

A clinical case is presented in which a patient with atrophic upper jaw is rehabilitated by a combination of zygomatic implants, placed using an exteriorized approach, and conventional palatalized anterior implants. A guided bone regeneration procedure is performed around the zygomatic implants to obtain a greater thickness of the facial bone and improve the prognosis. One year after loading, the peri-implant bone remains stable.

KEY WORDS: Zygomatic implants, guided bone regeneration, full arch, atrophic maxilla, fixed prosthesis.

Fecha de recepción: 16 de marzo de 2020

Fecha de aceptación: 25 de marzo de 2020

M. Peñarrocha Diago, J. Aizcorbe Vicente, M. Díaz Sánchez, B. Serra Pastor, D. Soto-Peñaloza, D. Peñarrocha Oltra. Regeneración ósea guiada simultánea en implantes cigomáticos con una aproximación exteriorizada en una atrofia maxilar avanzada. 2020; 36, (2): 63-70.

* Catedrático de Cirugía Bucal. Director del Master de Cirugía Oral e Implantología. Departamento de Cirugía Bucal. Facultad de Medicina y Odontología. Universitat de València.

* * Master Cirugía Oral e Implantología. Departamento de Cirugía Bucal. Facultad de Medicina y Odontología. Universitat de València.

*** Unidad de Oclusión y Prótesis. Facultad de Medicina y Odontología. Universitat de València.

**** Profesor Ayudante Doctor. Departamento de Cirugía Bucal. Facultad de Medicina y Odontología. Universitat de València. 


\section{INTRODUCCIÓN}

La rehabilitación mediante prótesis fijas implantosoportadas de los pacientes desdentados completos es un reto para el equipo odontológico. A pesar de que el número de estos pacientes ha disminuido en las últimas décadas en los países industrializados ${ }^{(1)}$, debido a una mejora en la calidad y un mayor acceso a la asistencia odontológica, todavía representan un importante sector de la población que requiere tratamiento ${ }^{(2)}$. Entre ellos, nos encontramos a un grupo, cada vez más importante, de pacientes que solicitan tratamiento tras el fracaso de una terapia implantológica previa. Todo ello, a hecho que cada vez sea más frecuente encontrarnos pacientes con un grado de atrofia severo, en donde, el tratamiento implantológico convencional se ve dificultado. Además, existe una presión creciente por los procedimientos de carga inmediata, tanto por los pacientes como por los profesionales, que evite los largos periodos de espera y devuelva rápidamente la función y la estética, especialmente en la mandíbula, donde las prótesis removibles convencionales se toleran mal.

Tradicionalmente, los pacientes atróficos requerían tratamientos regenerativos previos a la colocación de implantes. En muchos casos, la utilización de hueso autólogo de origen extraoral era la única opción ${ }^{(3)}$. Sin embargo, los largos periodos de espera entre las fases del tratamiento, así como la morbilidad asociada y los altos costes del tratamiento suponían que solo una mínima parte de los pacientes fueran candidatos a este tipo de terapia rehabilitadora. La introducción de los implantes cigomáticos en el mercado en $1999^{(4)}$ ha supuesto una revolución en el tratamiento de los pacientes atróficos maxilares. La fijación de estos implantes en el hueso cigomático, lejos del proceso alveolar, permite su utilización como anclaje para una prótesis fija, incluso en situaciones de atrofia extrema del maxilar superior. Solos o en combinación con implantes convencionales axiales o angulados permiten la rehabilitación de este tipo de pacientes en un periodo de tiempo menor, con mínima morbilidad, reduciendo los costes y en muchas ocasiones permitiendo protocolos de carga inmediata.

Se han descrito distintos abordajes para la colocación de los implantes cigomáticos ${ }^{(5)}$. La técnica clásica o intrasinusal $^{(4)}$, la técnica de la ranura sinusal ${ }^{(6)}$ y la téc- nica extrasinusal o exteriorizada ${ }^{(7)}$. De todas ellas, la técnica extrasinusal se ha asociado con un menor número de complicaciones sinusales (sinusitis maxilar) debido a la mínima o nula invasión del seno maxilar (8). Sin embargo, como consecuencia de esta técnica los implantes suelen quedar en una posición más exteriorizada que con las otras dos, lo que implica un menor grosor de la cortical vestibular, o directamente su ausencia, lo que puede comprometer el resultado del tratamiento a largo plazo.

El uso de substitutos óseos para el aumento de rebordes maxilares se considera hoy en día una alternativa predecible, aunque se sugiere el de una membrana de barrera para un resultado óptimo ${ }^{(9)}$. De especial interés en implantes cigomáticos, debido a que la calidad del reborde residual remanente alrededor de los implantes cigomáticos tiene un efecto sobre la distribución del estrés, y la presencia de defectos adicionales tiene un impacto negativo a este respecto ${ }^{(10)}$. Por lo tanto, en base a estar observaciones, el presente caso clínico muestra la técnica de regeneración ósea guiada alrededor de implantes cigomáticos colocados con la técnica extrasinusal para aumentar el grosor de la cortical vestibular y mejorar el pronóstico a largo plazo.

\section{CASO CLÍNICO}

\section{INFORMACIÓN DEL PACIENTE}

Se presenta el caso clínico de una paciente de 67 años de edad sin antecedentes sistémicos de interés, ni de hábito tabáquico, referida a la Unidad de Cirugía Bucal e Implantología. Departamento de Estomatología. Facultad de Medicina y Odontología. Universidad de Valencia.

\section{Hallazgos clínicos}

La paciente presenta el maxilar superior desdentado con atrofia severa y cuatro implantes con periimplantitis que soportan una sobredentadura inferior y desea una rehabilitación fija. Se observa que el plano oclusal inferior está muy elevado y la paciente casi no enseña los dientes superiores. Esto es debido a la presencia del síndrome combinado de Kelly (11), y a que en el momento de la colocación de los implantes inferiores no se tuvo esto en consideración. Este hecho hay que tenerlo en cuenta para la planificación del tratamiento de la mandíbula ya que habrá que realizar una ostectomía previa a la colocación de los implantes para situar el plano oclusal inferior más bajo y que la paciente 

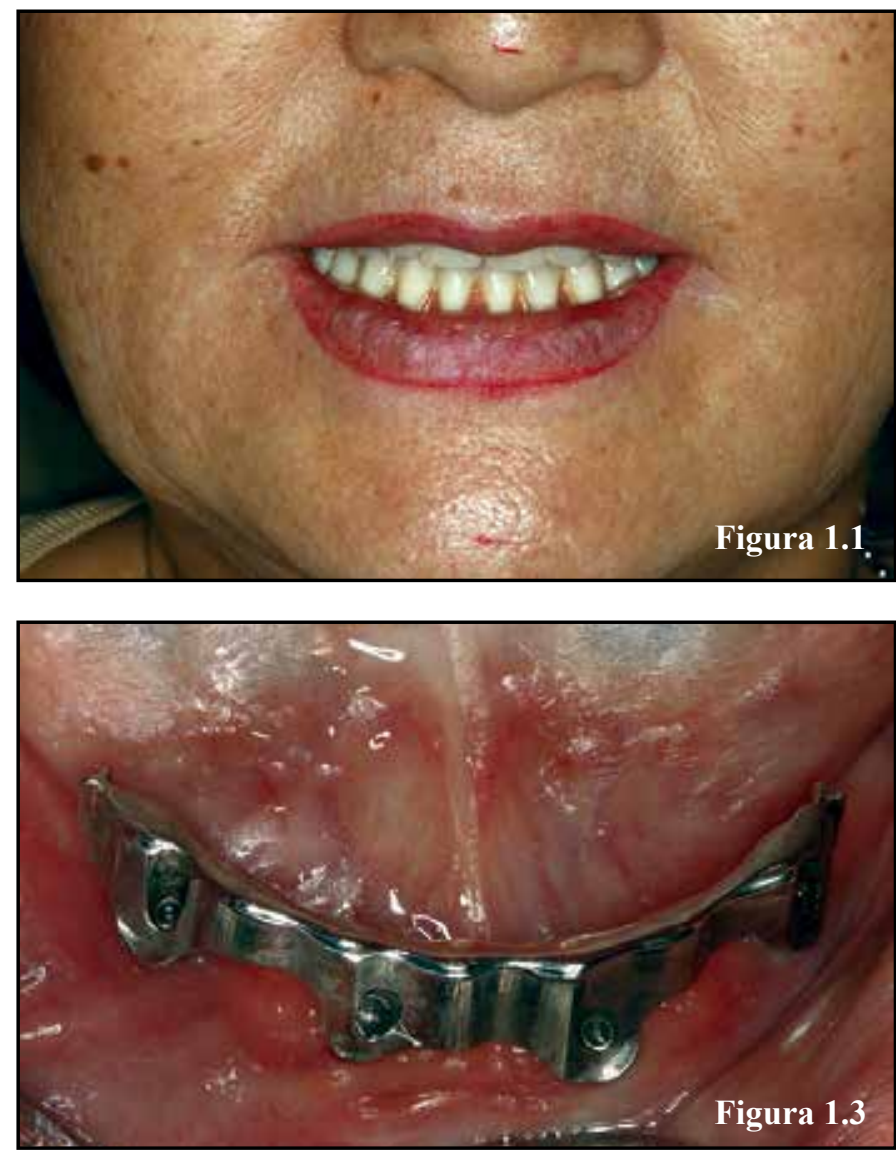

pueda mostrar los dientes superiores (fig. 1.1-1.4).

\section{Diagnóstico}

Una exploración del reborde óseo remanente de ambas arcadas se realiza mediante una tomografía computarizada de haz cónico (CBCT) Planmeca® system (Promax 3D Classic, Helsinki, Finland). La paciente presenta un grado de atrofia 6 en el maxilar posterior y 5 en el maxilar anterior, acorde a lo propuesto por Cawood and Howell ${ }^{(12)}$.

\section{INTERVENCIONES TERAPÉUTICAS}

Se decidió la colocación de dos implantes cigomáticos con técnica exteriorizada, y cuatro implantes axiales anteriores colocados palatinizados para una rehabilitación fija en el maxilar superior. En la mandíbula se planificó la explantación, ostectomía y colocación de cuatro implantes con carga inmediata (all-on-four) ${ }^{(13)}$.

La intervención se realizó empleando sedación consciente endovenosa realizada por una anestesista (Midazolam) y anestesia local infiltrativa con articaína con epinefrina 1:100.000 (Laboratorios Inibsa, Barcelona,
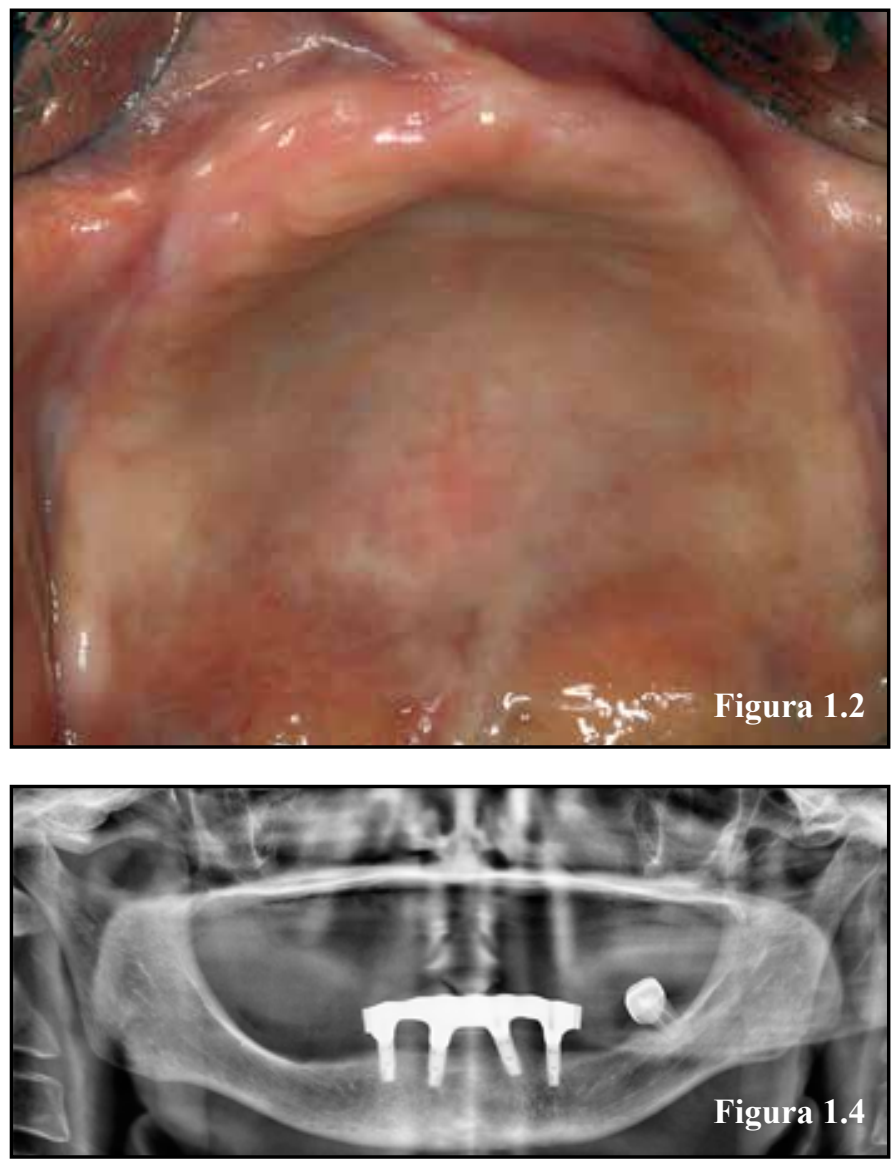

España). Tras una incisión crestal a lo largo del proceso edéntulo del maxilar superior, se realizaron dos incisiones de descarga a nivel del proceso cigomático. Se realizó un despegamiento a espesor completo y se localizaron las estructuras anatómicas relevantes: apertura piriforme, agujero infraorbitario y hueso cigomático. Una vez localizadas, se procedió a realizar la secuencia de fresado correspondiente para la colocación de dos implantes cigomáticos Galimplant IPX Smooth ${ }^{\circledR}$ (Galimplant ${ }^{\circ}$, Sarria, España) mediante la técnica exteriorizada o extrasinusal, procurando la mínima invasión del seno maxilar, e intentando conservar la mayor cantidad de cortical vestibular. Debido al grado de atrofia, se colocaron cuatro implantes convencionales Galimplant IPX® de conexión interna cónica palatinizados. Dos a nivel de la eminencia canina, y dos más anteriores, angulados mesialmente para buscar el hueso denso de la espina nasal anterior. Todos los implantes alcanzaron una adecuada estabilidad primaria (fig. 2.1). A continuación, se realizó un procedimiento de regeneración ósea guiada alrededor de todos los implantes con el objetivo de cubrir las espiras expuestas por palatino y aumentar el grosor de la cortical vestibular. El injerto óseo consistió en una mezcla 50:50 de hueso autólogo particulado obtenido de la 
AVANCES EN ODONTOESTOMATOLOGÍA

Vol. 36 - No 2 - 2020 Especial MonográFico IMPLANTOLOGÍA Oral
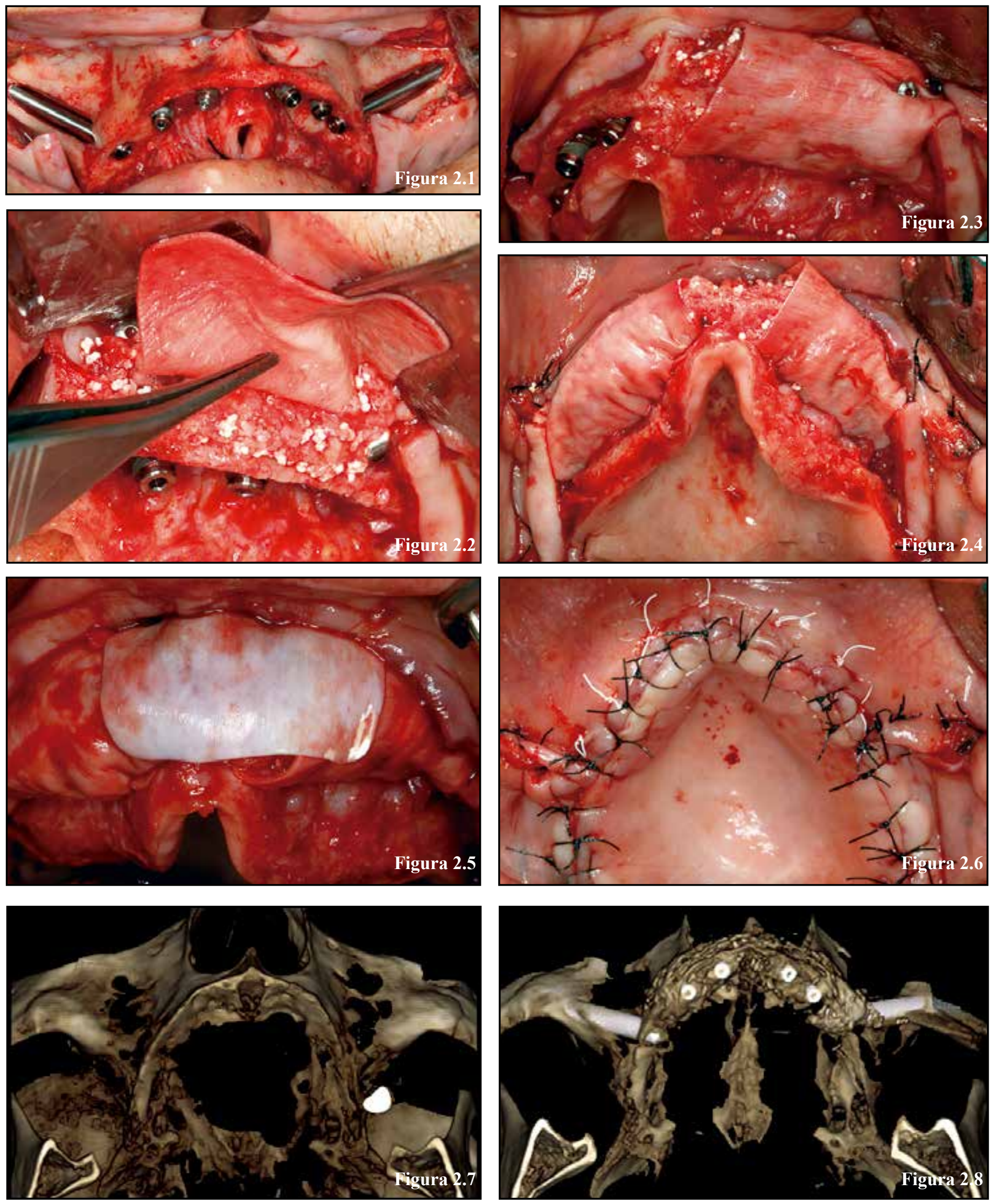
rama mandibular y un sustituto ósea aloplástico compuesto por ß-Fosfato Tricálcico (KeraOs®, Keramat, Santiago de Compostela, Galicia, España). El injerto óseo fue cubierto por membranas de colágeno porcino reabsorbibles (Creos Xenoprotect ${ }^{\circledR}$, Nobel Biocare, Gotenburgo, Suecia) fijadas mediante chinchetas (Meisinger pins) (Hager \& Mesisinger GmbH, Neuss, Germany) (figs. 2.2-2.2). Las membranas fueron tensadas y fijadas de tal manera que el material de injerto permanezca lo más estable posible (fig. 2.4, 2.5). El material de injerto sobrante fue colocado en la región más anterior del maxilar y cubierto con otra membrana de colágeno para obtener un mayor volumen óseo en esa región y mejorar el soporte labial. Para finalizar, se realizó una sutura en dos planos de los colgajos, mediante puntos colchoneros horizontales de aproximación de monofilamento 4/0 (PTFE, Cytoplast, Lubbock, Texas, EUA) y una segunda línea de puntos simples de cierre con material de sutura 4/0 (Supramid®, B. Braun, Rubí, Barcelona, Spain)(fig. 2.6). Las intervenciones fueron verificadas con una tomografía computarizada de haz cónico en el post-operatorio inmediato (figs. 2.7, 2.8).

Posteriormente, se realizó en la mandíbula un protocolo de all-on-four con carga inmediata tras la explantación de los implantes mientras que, los implantes del maxilar superior, quedaron sumergidos, portando una prótesis provisional superior de arcada completa.

\section{CUIDADOS POSTOPERATORIOS}

Como tratamiento postoperatorio, se le indicó a la paciente que tomara amoxicilina $500 \mathrm{mg}$ cada 8 horas durante 10 días y enjuagues de clorhexidina $0.12 \%, 3$ veces al día durante 10 días.

\section{SEgUIMIENTO Y RESULTADOS}

Tras un postoperatorio sin complicaciones, se realizó la segunda cirugía a los seis meses en el maxilar superior (fig. 3.1). Se realizó el levantamiento de los colgajos a espesor completo y se observó la integración del injerto particulado con un aumento significativo del volumen óseo, tanto en vestibular de los implantes cigomáticos como en el resto de la zona anterior y en los implantes por palatino, como se observa en las figuras 3.2 y 3.3 .

En la segunda fase quirúrgica, se atornillaron pilares transepiteliales para corregir las angulaciones de los implantes y seguidamente se suturaron los colgajos. Una vez, cicatrizaron los tejidos blandos (fig. 3.4). Pasadas dos semanas se tomaron impresiones de la ar-
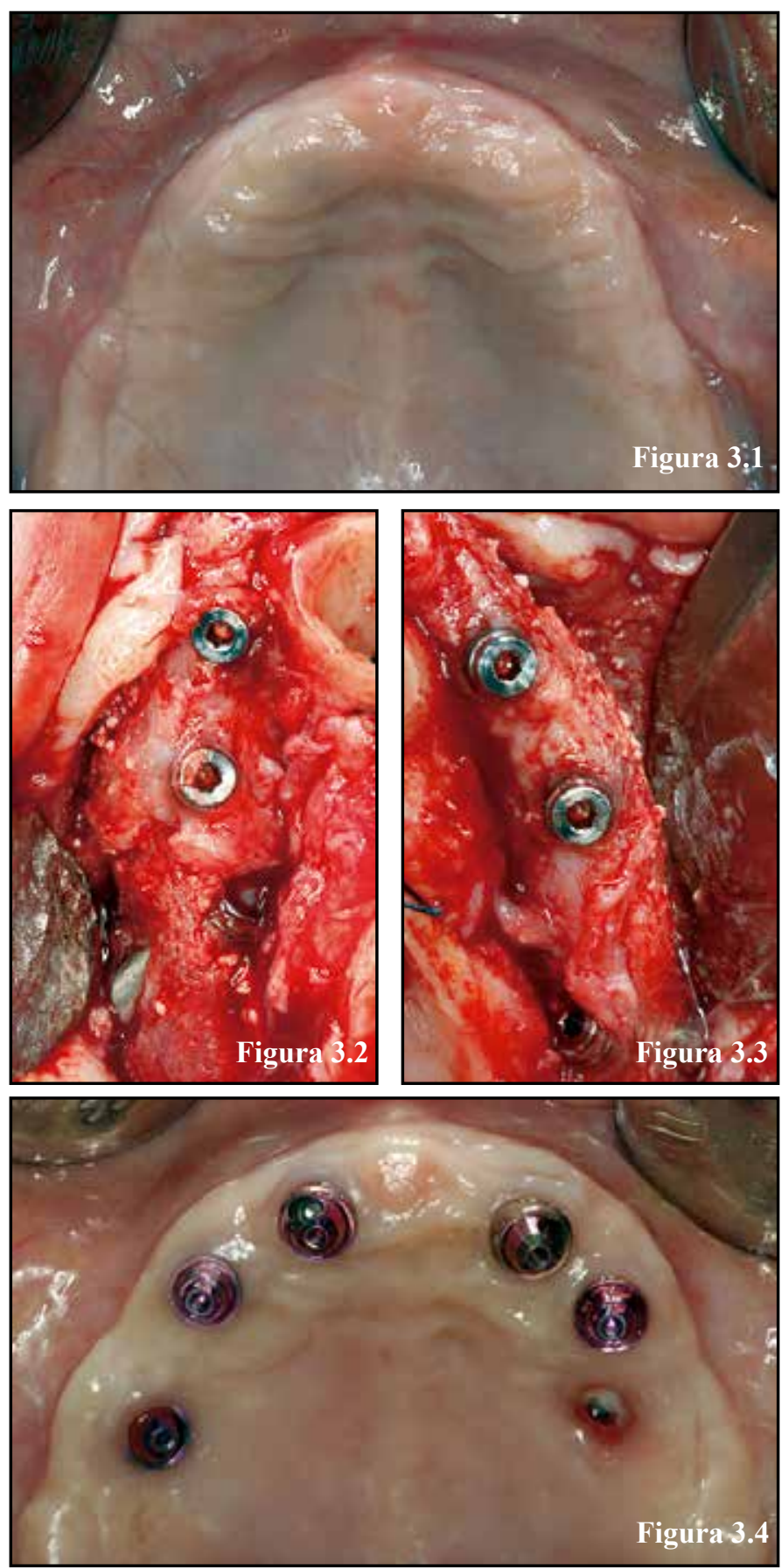

cada superior para la confección de una prótesis provisional que ayudó al modelado de los tejidos blandos. Tras 8 semanas se observó una correcta cicatrización y maduración de los tejidos blandos y se tomaron impresiones definitivas de ambas arcadas para la confección de 2 prótesis híbridas de titanio-resina (fig. 3.5 y 3.6). Al año de la carga protésica se realizó el control radiográfico con una ortopantomografía donde observa una estabilidad del hueso marginal peri-implantario como vemos en la figura 3.7. 

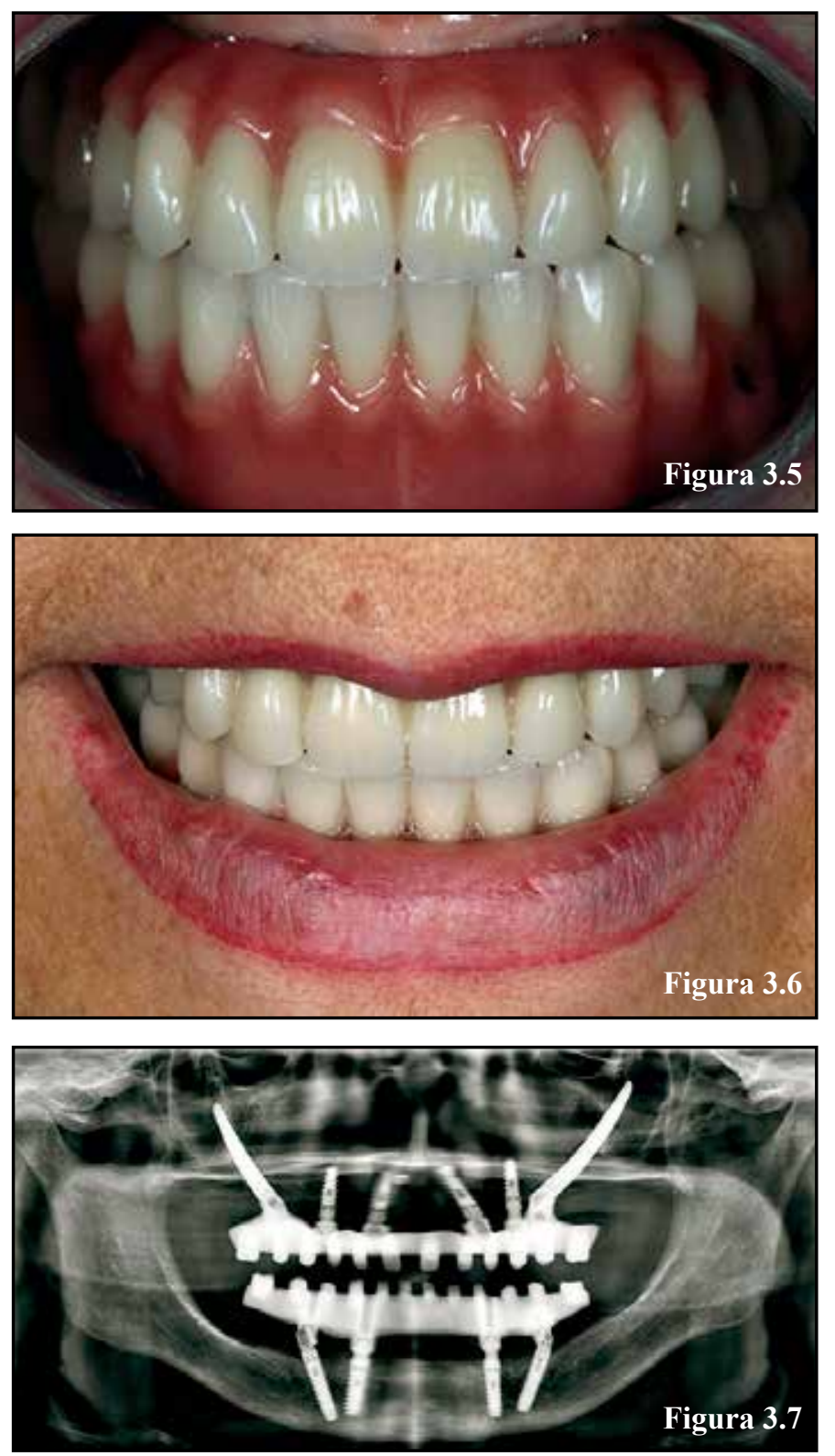

\section{DISCUSIÓN}

La utilización de implantes cigomáticos se ha demostrado como una técnica predecible y con altas tasas de éxito en el medio y largo plazo ${ }^{(14)}$, reduciendo de manera significativa el tiempo de tratamiento, la morbilidad y el coste ${ }^{(15)}$.

Aun siendo una técnica predecible, no está exenta de complicaciones, siendo la sinusitis maxilar la más frecuente $^{(8)}$. El riesgo de ocurrencia de sinusitis maxilar en relación a los implantes cigomáticos es de un $2.4 \%$ (8). Aunque su prevalencia puede verse infra-estimada debido a la poca información proporcionada y cortos periodos de seguimiento disponibles ${ }^{(8)}$. Sin embargo, parece existir un mayor riesgo de infecciones en el seno maxilar cuando se colocan implantes cigomáti$\cos ^{(16)}$ y dentales ${ }^{(17)}$ en pacientes previamente diagnosticados con sinusitis. Siendo dos factores, la ruptura de la membrana de schneider y la sinusitis previa los que aumentan en 2.86 y 21 veces el riesgo de sinusitis postoperatoria, respectivamente ${ }^{(18)}$.

Se han propuesto enfoques para evitar complicaciones, como el de la ventana ósea retenida tras una elevación sinusal extendida, la cual funciona a manera de escudo protegiendo la integridad de la membrana sinusal durante la inserción del implante cigomático, mostrando buenos resultados ${ }^{(19)}$. Por otro lado, el desarrollo de la técnica exteriorizada o extrasinusal ha supuesto un cambio de paradigma en la colocación de los implantes cigomáticos, reduciendo de manera significativa la ocurrencia de complicaciones sinusales maxilares debido a la conservación de la membrana de Schneider ${ }^{(20,21)}$.

Sin embargo, la presencia de una mínima cantidad de cortical vestibular o incluso su ausencia, es una consecuencia indeseable de esta técnica y que en nuestra opinión debe ser corregida mediante un injerto de tejidos duros o blandos en el momento de la colocación de los implantes cigomáticos. La presencia de una cortical vestibular gruesa mejora la estabilidad del hueso periimplantario a largo plazo.

Hoy en día no existe consenso sobre el espesor mínimo de hueso alveolar facial requerido para evitar posibles pérdidas ósea periimplantarias a largo plazo. Reportes previos como el de Spray y cols. (2000), sugieren un mínimo de $1.8-2 \mathrm{~mm}$ es requerido para disminuir la pérdida ósea vertical ${ }^{(22)}$. Un trabajo preclínico realizado en perros por Monje y cols. (2919), comparan sitios de implantación con $>1.5 \mathrm{~mm} 0<1.5$. mm respecto a la pérdida ósea vertical ${ }^{(23)}$. Encontrando que aquellos sitios con grosor $\geq 1.5 \mathrm{~mm}$ mostraron significativamente menos pérdida ósea en sentido vertical y horizontal que los sitios con $<1.5 \mathrm{~mm}^{(23)}$.

Por lo tanto, no hay duda de que mantener una dimensión de la cortical bucal no sólo nos ayudará a mantener los tejidos periimplantarios a largo plazo, sino que también ayuda a una adecuada distribución del estrés. Ya que la presencia de defectos óseos alrededor de implantes cigomáticos tiene un impacto negativo negativo en la distribución del estrés ${ }^{(24)}$. Además, cabe mencionar que la distribución de cargas en el sector posterior del 
maxilar se involucra de forma similar entre el hueso trabeculado y el componente cortical; opuesto a lo que ocurre en la zona anterior de la maxila donde la cortical es quien asimila y distribuye mejor el estrés ante la magnitud y dirección de las fuerzas oclusales ${ }^{(25)}$.

Para este propósito, la técnica de regeneración ósea guiada se ha mostrado efectiva alrededor de implantes convencionales en el maxilar superior a largo plazo ${ }^{(26)}$. Un estudio de cohorte reciente, observó que la reabsorción ósea y recesión de tejidos blandos periimplantarios fue masiva cuando el espesor del hueso alveolar facial fue menor a $0.5 \mathrm{~mm}$ a un año de seguimiento, comparado al grupo con valores $\geq 0.5 \mathrm{~mm}^{(27)}$.

La novedad del presente enfoque, se debe que según el conocimiento de los autores este es uno de los primeros reportes de caso en el que se utiliza la técnica de regeneración ósea guiada alrededor de los implantes cigomáticos para aumentar el grosor de la cortical vestibular, previniendo así la recesión de los tejidos blandos por vestibular, un factor que puede mejorar el pronóstico a largo plazo. Sin embargo, un mayor seguimiento y número de casos son necesarios, por lo que futuros estudios a este respecto están garantizados.

\section{CONCLUSIONES}

La regeneración ósea guiada alrededor de implantes cigomáticos es una técnica que incrementa el grosor de la cortical vestibular, previene la recesión de tejidos blandos y se mantiene estable en el corto plazo. Además, la técnica exteriorizada intenta prevenir la sinusitis maxilar, una complicación muy frecuente en la cirugía de implantes cigomáticos.

\section{BIBLIOGRAFIA}

1. Slade GD, Akinkugbe AA, Sanders AE. Projections of U.S. edentulism prevalence following 5 decades of decline. J Dent Res, 2014; 93:959-965

2. Emami E, Freitas de Souza R, Kabawat M, Feine JS. The impact of edentulism on oral and general health. Int J Dent, 2013: 498305

3. Keller EE, Tolman DE, Eckert SE. Maxillary antral-nasal inlay autogenous bone graft reconstruction of compromised maxilla: A 12- year retrospective study. Int $\mathrm{J}$ Oral Maxillofac Implants 1999; 14:707-721

4. Brånemark PI, Gröndahl K, Ohrnell LO, Nilsson P, Petruson B, et al. Zygoma fixture in the management of advanced atrophy of the maxilla: technique and long-term results. Scand J Plast Reconstr Surg Hand Surg 2004; 38:70-85

5. Migliorança RM, Irschlinger AL, Peñarrocha-Diago M, Fabris RR, Aizcorbe-Vicente J, Zotarelli Filho IJ. History of zygomatic implants: A systematic review and meta-analysis. Dent Oral Craniofac Res 2019; 5:1-9

6. Stella JP, Warner MR. Sinus slot technique for simplification and improved orientation of zygomaticus dental implants: A technical note. Int J Oral Maxillofac Implants 2000; 15:889-893

7. Migliorança RM, Ilg JP, Mayo TM, Serrano AS, Funis LP, et al. Função imediata em fixações zigomáticas: relato de dois casos com 18 e 30 meses de acompanhamento clínico. Implant News 2006; 3:243-247

8. Chrcanovic BR, Albrektsson T, Wennerberg A. Survival and complications of zygomatic implants: An updated systematic review. J Oral Maxillofac Surg 2016; 74:1949- 1964.

9. Thoma DS, Bienz SP, Figuero E, Jung RE, Sanz-Martín I. Efficacy of lateral bone augmentation performed simultaneously with dental implant placement: A systematic review and meta-analysis. J Clin Periodontol. 2019 Jun;46 Suppl 21:257-276.

10. Duan Y, Chandran R, Cherry D. Influence of Alveolar Bone Defects on the Stress Distribution in Quad Zygomatic Implant-Supported Maxillary Prosthesis. Int J Oral Maxillofac Implants. 2018 May/Jun;33(3):693-700.

11. Kelly E. Changes caused by a mandibular removable partial denture opposing a maxillary complete denture. J Prosthet Dent. 1972 Feb;27(2):140-50.

12. Cawood JI, Howell RA. A classification of the edentulous jaws. Int J Oral Maxillofac Surg. 1988 Aug;17(4):232-6.

13. Soto-Penaloza D, Zaragozí-Alonso R, Penarro- 
cha-Diago M, Penarrocha-Diago M. The allon-four treatment concept: Systematic review. J Clin Exp Dent. 2017 Mar 1;9(3):e474-e488. doi: $10.4317 /$ jced.53613.

14. Alzoubi F, Bedrossian E, Wong A, Farrell D, Park C, Indresano T. Outcomes assessment of treating completely edentulous patients with a fixed implant-supported profile prosthesis utilizing a graftless approach. Part 1: Clinically related outcomes. Int $\mathrm{J}$ Oral Maxillofac Implants 2017; 32:897-903.

15. Davó R, Felice P, Pistilli R, Barausse C, Marti-Pages C, et al. Immediately loaded zygomatic implants vs conventional dental implants in augmented atrophic maxillae: 1-year postloading results from a multicenter randomised controlled trial. Eur J Oral Implantol 2018; 11:145-161.

16. Malo P, de Araujo Nobre M, Lopes A, et al: Extramaxillary surgical technique: Clinical outcome of 352 patients rehabilitated with 747 zygomatic implants with a follow-up between 6 months and 7 years. Clin Implant Dent Relat Res 2015; 17(suppl 1):e153, 2015

17. Kozuma A, Sasaki M, Seki K, Toyoshima T, Nakano H, Mori Y. Preoperative chronic sinusitis as significant cause of postoperative infection and Implant loss after sinus augmentation from a lateral approach. Oral Maxillofac Surg. 2017 Jun;21(2):193-200.

18. Kim JS, Choi SM, Yoon JH, Lee EJ, Yoon J, Kwon SH, Yeo CD, Ryu JS, Lee JH, You YS, Kim SG, Lee MH, Han BH. What Affects Postoperative Sinusitis and Implant Failure after Dental Implant: A Meta-analysis. Otolaryngol Head Neck Surg. 2019 Jun;160(6):974-984.

19. Chow J, Wat P, Hui E, Lee P, Li W. A new method to eliminate the risk of maxillary sinusitis with zygomatic implants. Int J Oral Maxillofac Implants. 2010 Nov-Dec;25(6):1233-40.

20. Miglioranza R, Sotto-Maior B, Senna P, Francischone C, Del Bel Cury A: Immediate occlusal loading of extrasinus zygomatic implants: A prospective cohort study with a follow-up period of 8 years. Int. J. Oral Maxillofac. Surg. 2012.
21. Migliorança RM, Coppedê A, Dias Rezende $\mathrm{RC}$, de Mayo T. Restoration of the edentulous maxilla using extrasinus zygomatic implants combined with anterior conventional implants: a retrospective study. Int $\mathrm{J}$ Oral Maxillofac Implants. 2011;26:665-72.

22. Spray JR, Black CG, Morris HF, Ochi S. The influence of bone thickness on facial marginal bone response: stage 1 placement through stage 2 uncovering. Ann Periodontol. 2000 Dec;5(1):119-28.

23. Monje A, Chappuis V, Monje F, Muñoz F, Wang HL, Urban IA, Buser D. Critical Peri-implant Buccal Bone Wall Thickness Revisited: An Experimental Study in the Beagle Dog. Int J Oral Maxillofac Implants. 2019 Sep 18.

24. Duan Y, Chandran R, Cherry D. Influence of Alveolar Bone Defects on the Stress Distribution in Quad Zygomatic Implant-Supported Maxillary Prosthesis. Int J Oral Maxillofac Implants. 2018 May/Jun;33(3):693-700.

25. Janovic A, Saveljic I, Vukicevic A, et al (2015) Occlusal load distribution through the cortical and trabecular bone of the human mid-facial skeleton in natural dentition: A three-dimensional finite element study. Ann Anat - Anat Anzeiger 197:16-23. doi: 10.1016/j.aanat.2014.09.002

26. Buser D, Chappuis V, Kuchler U, Bornstein MM, Wittneben JG, Buser R, Cavusoglu Y, Belser UC. Long-term stability of early implant placement with contour augmentation. J Dent Res 2013; 92 (Suppl. 2):176S-182S.

27. Yang X, Zhou T, Zhou N, Man Y (2019) The thickness of labial bone affects the esthetics of immediate implant placement and provisionalization in the esthetic zone: A prospective cohort study. Clin Implant Dent Relat Res 21(3):482-491. doi: 10.1111/cid.12785

\section{AUTOR DE CORRESPONDENCIA:}

David Peñarrocha Oltra

Departamento de Estomatología

Universitat de València

C/ Gascó Oliag 1,

46010 - Valencia (España)

E-mail: david.penarrocha@uv. 